\title{
Effect of Phase Angle on Fatigue Failure of 30CrMnSiA Steel under Tension-torsion Fatigue Loading
}

\author{
Tianqi Liu ${ }^{1}$, Xinhong Shi ${ }^{1}$ and Jianyu Zhang ${ }^{2}$ \\ ${ }^{1}$ School of Aeronautic Science and Engineering, Beijing University of Aeronautics and Astronautics, Beijing 100191, China. \\ ${ }^{2}$ School of Aeronautics and Astronautics, Chongqing University, Chongqing 400044, China.
}

\begin{abstract}
Tension-torsion high-cycle fatigue experiments with solid cylindrical bar specimens of 30CrMnSiA steel were carried out under different phase angles. And the experiment data, characteristics of stresses with plane orientation and fracture appearance under different loading conditions were analyzed and investigated. Experiment results show that fatigue life increases gradually with the increase of phase angle. The fatigue life is shortest for $0^{\circ}$ phase angle, while the fatigue life is longest for $90^{\circ}$ out-of-phase angle. It could be found from analysis of stress parameters that as the phase angle increases, the shear stress amplitude decreases and the maximum normal stress increases gradually, which are on the maximum shear stress amplitude plane close to the axial of specimen. Macro-analysis of the fracture appearances show that when the phase angle increases, the angel between crack initiation plane and specimen axial decreases, and cracks are inclined to initial and propagate on or near the maximum shear stress amplitude plane with larger maximum normal stress under proportional loading as well as non-proportional loading.
\end{abstract}

\section{Introduction}

$30 \mathrm{CrMnSiA}$ steel is a kind of quenched and tempered steel with the advantages of high strength and fatigue resistance that widely used in aircraft, such as the landing gear, engine mount and important bolt structure and components. The structure and components in practical engineering is often under multiaxial fatigue load. A large number of studies have shown that the phase angle is an important parameter in the study of multiaxial fatigue.

For the influence of phase difference on fatigue failure, McDiarmid [1,2] analysed the stress characteristics of different load cases and assumed that the existence of a phase angle will have a positive effect on the fatigue failure. Papadopoulos [3,4] assumed that the existence of a phase angle has little influence on hard metals, but for ductile metals, the influence of the phase angle on fatigue failure cannot be ignored. Susmel $[5,6]$ thought that the existence of phase has a negative effect on the fatigue failure of ductile metals. Tovo[7] studied the fatigue failure of EN-GJS-400-15 cast iron and found that the multiaxial fatigue strength increases when phase angle exists. Anes [8] found that the existence of a phase angle has a negative effect on multiaxial fatigue failure of 42CrMo4 steel and AZ31B-F Mg alloy. Shi [9] found that the existence of a phase angle has a positive effect on the fatigue life of 2A12-T4 aluminum alloy under tension-torsion fatigue loading.
To investigate the effects of phase difference on the tension-torsion, high-cycle fatigue failure of $30 \mathrm{CrMnSiA}$ steel, both experiments and theoretical analysis are conducted in this paper.

\section{Experiments}

\subsection{Material and specimens}

The material used in the current experimental investigation is $30 \mathrm{CrMnSiA}$ structure steel. The chemical compositions of the $30 \mathrm{CrMnSiA}$ structure steel are: $\mathrm{C}(0.31 \%), \operatorname{Mn}(0.85 \%), \mathrm{Si}(0.99 \%), \mathrm{S}(0.009 \%), \mathrm{P}(0.01 \%)$, $\mathrm{Ni}(0.05 \%), \quad \mathrm{Cr}(0.87 \%), \quad \mathrm{W}(0.01 \%), \quad \mathrm{Mo}(0.02 \%)$, $\mathrm{V}(0.01 \%), \mathrm{Cu}(0.18 \%), \mathrm{Ti}(0.003 \%), \mathrm{Fe}$ (the rest). The tension elastic modulus is $207 \mathrm{GPa}$, tension yield strength is $1196 \mathrm{MPa}$, and tension strength is $1334 \mathrm{MPa}$. The torsion modulus is $77.2 \mathrm{GPa}$, torsion yield strength is $825 \mathrm{MPa}$, and torsion strength is $1040 \mathrm{MPa}$.

The solid cylindrical bar specimen is adopted, which machined from the round bar of $\Phi=30 \mathrm{~mm}$. The minimum diameter of $30 \mathrm{CrMnSiA}$ steel specimen is $\mathrm{D}=12 \mathrm{~mm}$. All of the specimens are polished with several abrasive papers up to grade 2000. The dimension tolerance is $0.02 \mathrm{~mm}$ and surface finish of the specimen is less than $0.2 \mu \mathrm{m}$ to reduce the scatter of the experiment result. The knuckle radius of the specimen is $\mathrm{R}=220 \mathrm{~mm}$. Shape and dimensions of the specimen is shown in Fig. 1. 


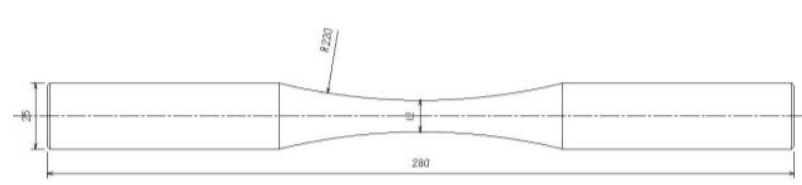

Figure 1. Shape and dimensions of the specimen.

\subsection{Experiment procedure}

The fatigue experiments of this study are performed on a PLS-200/1500 servo-hydraulic tension-torsion load frame. The test system, which has a capacity of $1500 \mathrm{Nm}$ in torque and $200 \mathrm{kN}$ in axial load, is equipped with electronic and computer controls, as well as data acquisition. The axial and shear loads are controlled concurrently, while the cyclic loading is fully reversed. All of the experiments are conducted at room temperature in ambient air. The loading frequency is $\mathrm{f}=3 \mathrm{~Hz}$ in the experiments, and in each load case, three or four specimens have been tested. The waveform of the load is described as follows:

$$
\begin{gathered}
\sigma_{\mathrm{x}}=\sigma_{\mathrm{x}, \mathrm{a}} \sin \omega t \\
\tau_{\mathrm{xy}}=\tau_{\mathrm{xy}, \mathrm{a}} \sin (\omega t-\delta)
\end{gathered}
$$

where $\sigma_{\mathrm{x}}$ is the axial stress, $\sigma_{\mathrm{x}, \mathrm{a}}$ is the axial stress amplitude; $\tau_{\mathrm{xy}}$ is the shear stress, $\tau_{\mathrm{xy}, \mathrm{a}}$ is torsion stress amplitude; and $\delta$ is the phase angle between tension stress and torsion stress. Both the axial stress amplitude and shear stress amplitude are $357.5 \mathrm{MPa}$ in the test, and four different phase angles $\left(\delta=0^{\circ}, 30^{\circ}, 60^{\circ}, 90^{\circ}\right)$ are chosen for the test. The loading paths are shown in Fig. 2.

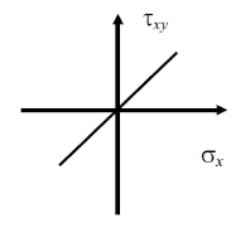

(a) $\delta=0^{\circ}$

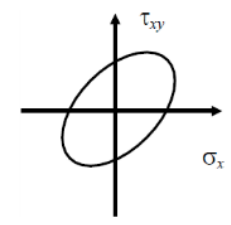

(c) $\delta=60^{\circ}$

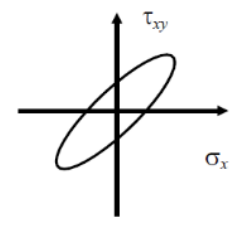

(b) $\delta=30^{\circ}$

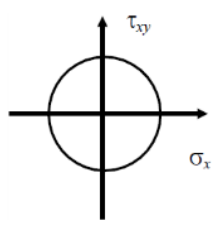

(d) $\delta=90^{\circ}$
Figure 2. Loading paths for the tension-torsion fatigue tests with different phase angles.

\section{Results and discussion}

\subsection{Fatigue life}

Table 1 shows the median fatigue life of $30 \mathrm{CrMnSiA}$ steel under different phase angles, and the tension-torsion fatigue test results are shown in Figure 3. In the figure, tension-torsion fatigue lives of specimens are described by the open label, and the solid label means the median life in each load case. It can be seen from the figure that the median fatigue life increases with the increase of phase angle. Therefore, the existence of a phase angle has a positive effect on the fatigue life of $30 \mathrm{CrMnSiA}$ steel, which is same to 2A12-T4 aluminum alloy [9]. The difference between the two materials is that the fatigue life of 2A12-T4 aluminum alloy increased about three times when the phase angle increased from $0^{\circ}$ to $90^{\circ}$, but for $30 \mathrm{CrMnSiA}$ steel, fatigue life increased only about 1.3 times when the phase angle increased from $0^{\circ}$ to $90^{\circ}$. This may because that the plastic flow performance of 2A12-T4 aluminum alloy is better than $30 \mathrm{CrMnSiA}$ steel [10].

Tabel 1. Fatigue life under different phase angles.

\begin{tabular}{|c|c|c|c|c|}
\hline $\boldsymbol{\delta} /{ }^{\circ}$ & $\mathbf{0}$ & $\mathbf{3 0}$ & $\mathbf{6 0}$ & $\mathbf{9 0}$ \\
\hline fatigue life $/ \times 10^{5}$ & 1.02 & 1.09 & 1.26 & 1.30 \\
\hline
\end{tabular}

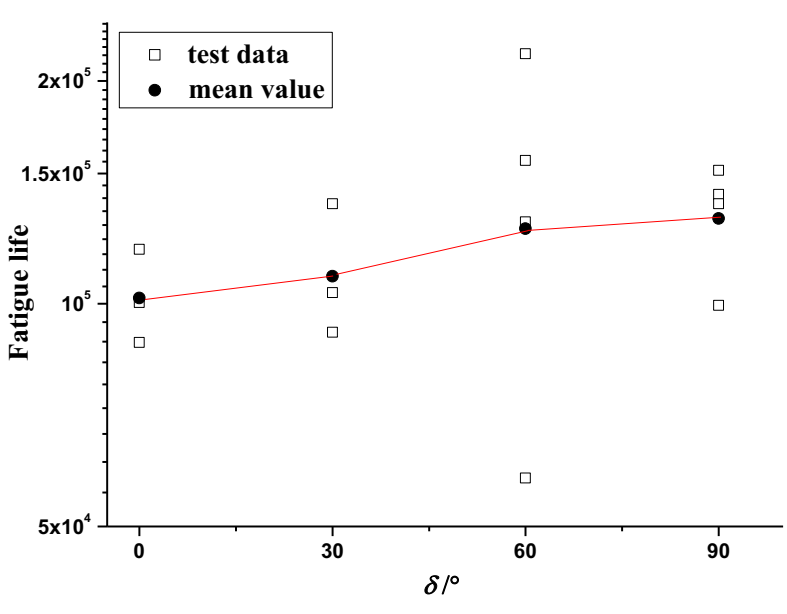

Figure 3. Relation of fatigue life and $\delta$

\subsection{Stress analysis}

Fig. 4(a) shows the loading forms on the specimen. The $\mathrm{X}$ axis is defined as the longitudinal direction of the specimen and $\mathrm{Y}$ axis is perpendicular to $\mathrm{X}$ axis. The angle between an arbitrary plane and the $\mathrm{X}$ axis is defined as $\varphi$.

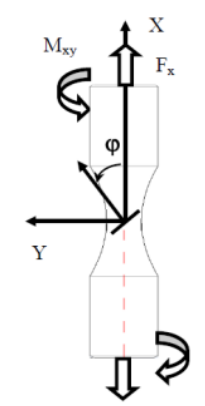

(a)

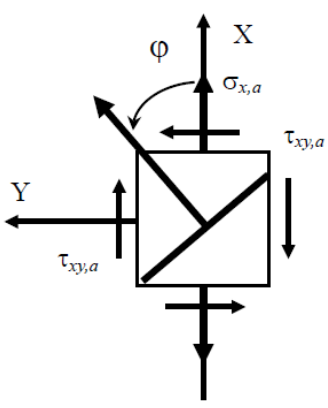

(b)
Figure 4. Stress analysis

It is widely believed that crack initiation is mainly controlled by the amplitude of alternating shear stress. Due to the derivation of Papadopoulos [4], the maximum shear stress amplitude is on the planes that perpendicular 
to the XY plane. For the specimens under tension-torsion fatigue load, the critical point is on the surface of the specimen. Fig. 4(b) shows the stress element on the surface of the specimen. The changes of shear stress amplitude with different phase angles on different planes are shown in Fig. 5. It can be seen from the figure that two maximum shear stress amplitude planes exist for each load case. The maximum normal stress on different planes is shown in Fig. 6.

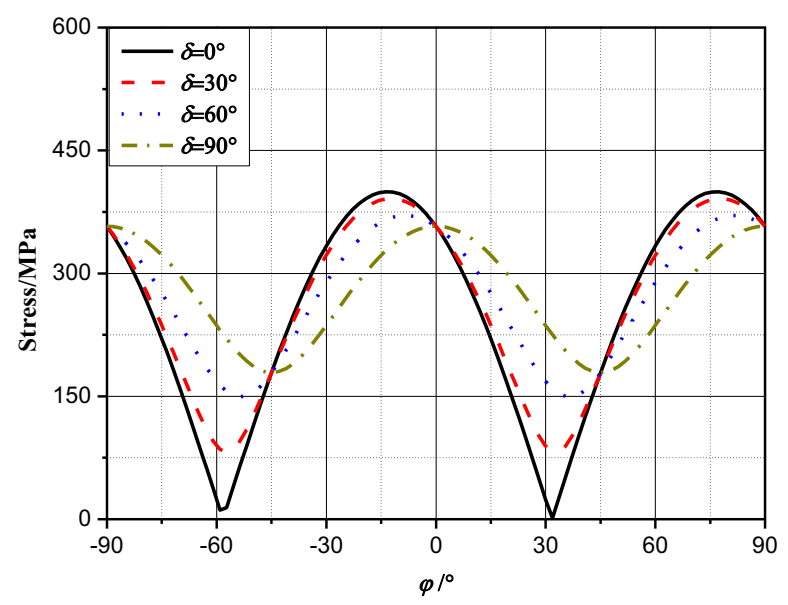

Figure 5.Variations of shear stress amplitude on different planes

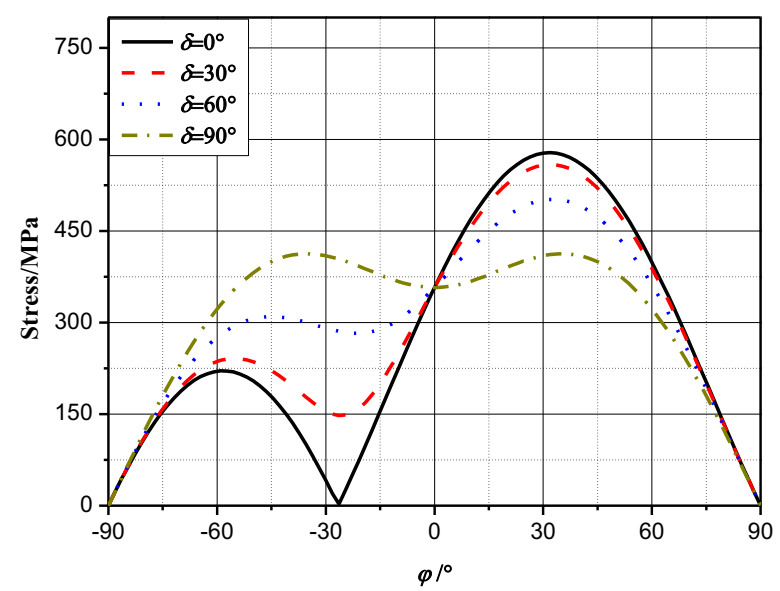

Figure 6.Variations of maximum normal stress on different planes

Fig. 7 shows the change of the maximum shear stress amplitude and the two maximum normal stresses on the maximum shear stress amplitude planes under different phase angles. The values of the stress parameters are given in Table 2 when the phase angle $\delta=0^{\circ}, 30^{\circ}, 60^{\circ}, 90^{\circ}$ respectively.

The two maximum normal stresses on the two maximum shear stress amplitude planes are defined as $\sigma_{\mathrm{n} 1, \max }$ and $\sigma_{\mathrm{n} 2, \max }$, respectively. It can be seen that the maximum shear stress amplitude $\left(\tau_{n, \max }\right)$ decreases from $399.7 \mathrm{MPa}$ to $357.5 \mathrm{MPa}$ when the phase angle increases from $0^{\circ}$ to $90^{\circ}$. The maximum normal stress $\sigma_{\mathrm{n} 1 \text {, max }}$ increases with the increase of phase angle while $\sigma_{\mathrm{n} 2 \text {,max }}$ decreases. Therefore, as the fatigue life increases with the increase of phase angle, the maximum shear stress amplitude may be the main cause of fatigue failure for 30CrMnSiA steel under tension-torsion fatigue loading.

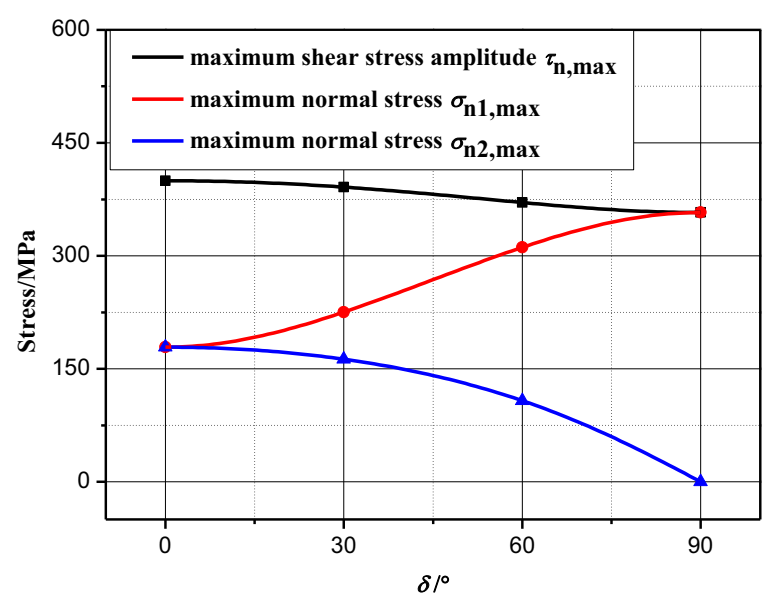

Figure 7.Variations of maximum shear stress amplitude and maximum normal stresses under different phase angles

Table 2. Directions of the maximum shear stress amplitude and the crack initiation plane.

\begin{tabular}{|c|c|c|c|c|}
\hline $\boldsymbol{\delta} /{ }^{\circ}$ & $\mathbf{0}$ & $\mathbf{3 0}$ & $\mathbf{6 0}$ & $\mathbf{9 0}$ \\
\hline$\tau_{\mathrm{n}, \max } / \mathrm{MPa}$ & 399.7 & 391.27 & 370.78 & 357.5 \\
\hline$\sigma_{\mathrm{n} 1, \max } / \mathrm{MPa}$ & 178.75 & 225.28 & 311.24 & 357.5 \\
\hline$\sigma_{\mathrm{n} 2, \max } / \mathrm{MPa}$ & 178.75 & 162.75 & 107.64 & 0 \\
\hline
\end{tabular}

\subsection{Fracture appearance}

The fracture appearances of the $30 \mathrm{CrMnSiA}$ steel specimens under tension-torsion fatigue loading at different phase angles are shown in Fig. 8.

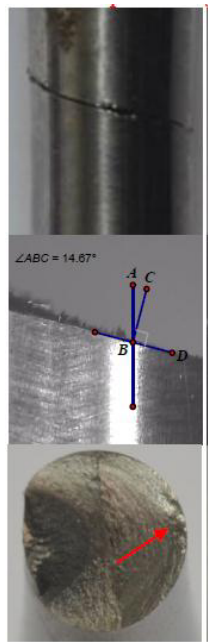

(a) $\delta=0^{\circ}$

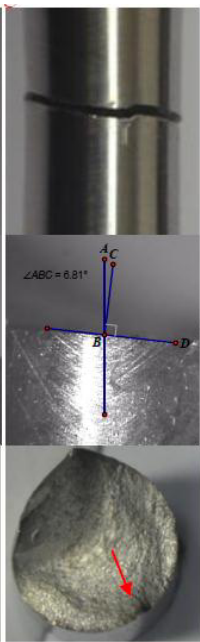

(b) $\delta=30^{\circ}$

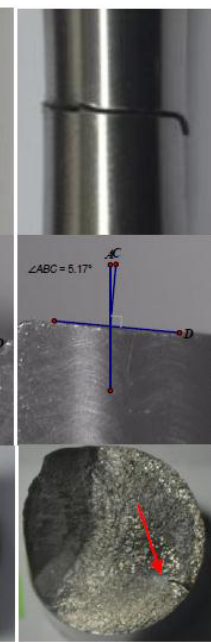

(c) $\delta=60^{\circ}$

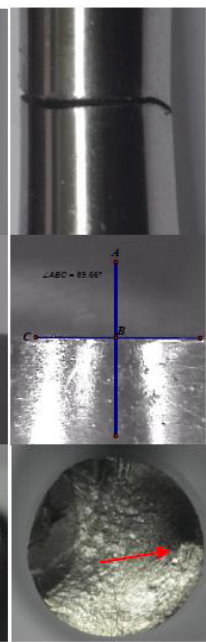

(d) $\delta=90^{\circ}$
Figure 8. Early crack propagation direction and fracture appearance under different phase angles

It can be seen from the figure that crack initiated on the surface of the specimens as indicated by a red arrow. The extension and final regions can be clearly observed. As 
the phase angle increases, the direction of the crack on the surface of the specimen gradually tends to become perpendicular to the axial of the specimen. The crack initiation angle is similar to the direction of the maximum shear stress amplitude plane and gradually flattens as the phase angle increases. The appearances of the fractures are smooth and the areas of the final rupture regions are nearly identical at different phase angles observed.

Table 3 shows the theoretical directions of the maximum shear stress amplitude plane $(\varphi)$ and measured angle of the crack initiation plane $\left(\varphi^{*}\right)$ under tensiontorsion load with different phase angles.

Table 3. Directions of the maximum shear stress amplitude and the crack initiation plane.

\begin{tabular}{|c|c|c|c|c|}
\hline $\boldsymbol{\delta} /{ }^{\circ}$ & $\mathbf{0}$ & $\mathbf{3 0}$ & $\mathbf{6 0}$ & $\mathbf{9 0}$ \\
\hline $\boldsymbol{\varphi} /{ }^{\circ}$ & 76.7 & 77.7 & 81.6 & 90 \\
\hline $\boldsymbol{*} * / \circ$ & -13.3 & -12.3 & -8.4 & 0 \\
\hline
\end{tabular}

When $\delta=0^{\circ}$, which is shown in Fig. $8(\mathrm{a})$, the crack initiated on the surface of the specimen, and the initial crack is in the direction of maximum shear stress amplitude $\left(-13.3^{\circ}\right)$ with a higher maximum normal stress, as shown in the previous analysis. The propagation of the surface crack is approximately along the same direction until failure occurs. The fracture appearance is smooth, and shear characteristics are clearly shown. When $\delta=30^{\circ}$ and $\delta=60^{\circ}$, which are both shown in Figs. 8(b) and 8(c), respectively, the angles between the initial cracks and the axial of the specimens decrease, and the fracture appearances become flatten. Similarly, the initial crack is in the direction of maximum shear stress amplitude plane with a higher maximum normal stress. When $\delta=90^{\circ}$, which is shown in Fig. 8(d), the maximum shear stress is shown to be perpendicular to the axial direction of the specimen in this case, the crack initiated in the same direction as the maximum shear stress. The fracture appearance is also nearly perpendicular to the axial direction of the specimen.

Based on the discussion above, test results show that the fatigue life increases with the increase of phase angle. The maximum shear stress amplitude decreases with the increase of phase angle. The crack initiation and early propagation direction is close to the maximum shear stress amplitude plane with a higher maximum normal stress. Therefore, both the maximum shear stress amplitude and the maximum normal stress on the maximum shear stress amplitude plane effect the fatigue failure of $30 \mathrm{CrMnSiA}$ steel under tension-torsion fatigue loading at different phase angles, and the maximum shear stress amplitude seems to be the dominant factor.

\section{Conclusions}

The tension-torsion fatigue tests for $30 \mathrm{CrMnSiA}$ steel show that:

(1) with the increase of phase angle, fatigue life of the $30 \mathrm{CrMnSiA}$ steel increases, which means the fatigue life is shortest under proportional loading $\left(\delta=0^{\circ}\right)$ and longest when the phase angle $\delta=90^{\circ}$;

(2) the maximum shear stress is known to be the dominant factor that causes fatigue failure in $30 \mathrm{CrMnSiA}$ steel under tension-torsion fatigue loading at different phase angles;

(3) with the increase of phase angle, the angle between the initial crack plane and the longitudinal of the specimen decreases, and the crack initiation and early propagation is usually along the maximum shear stress amplitude plane with a greater maximum normal stress.

\section{Acknowledgement}

The National Natural Science Foundation of China is acknowledged for supporting the project (11172021).

\section{References}

1. D. L. McDiarmid, Fatig Fract Eng Mater Struct 9.6, 457-475 (1987).

2. D. L. McDiarmid, Fatig Fract Eng Mater Struct 14.4, 429-453 (1990).

3. I. V. Papadopoulos, Fatig Fract Eng Mater Struct 18.1, 79-91 (1995).

4. I. V. Papadopoulos, P. Davoli, C. Gorla, M. Filippini, A. Bernasconi, Int J Fatigue 19.3, 219-235 (1997).

5. L. Susmel, N. Petrone, European Structural Integrity Society 31.03, 83-104 (2003).

6. L. Susmel, R. Tovo, P. Lazzarin, Int J Fatigue 27, 928-943 (2005).

7. R. Tovo, P. Lazzarin, F. Berto, M. Cova, E. Maggiolini, Theor Appl Fract Mec 73, 60-67 (2014).

8. V. Anes, L. Reis, B. Li, M. Freitas, Int J Fatigue 58, 102-113 (2014).

9. X. H. Shi, J. Y. Zhang, R. Bao, Journal of Aeronautical Materials 36.8, 965-968 (2010).

10. C. C. Zhang, Fatigue life prediction of structures in HCF region under complex stress field (2010). 